\title{
Exchange Perturbation Theory for Multiatomic Electron System and Its Application to Spin Arrangement in Manganite Chains
}

\author{
E. V. Orlenko, ${ }^{1}$ T. S. Orlova, ${ }^{2}$ F. E. Orlenko, ${ }^{1}$ and G. G. Zegrya ${ }^{1,2}$ \\ ${ }^{1}$ Theoretical Physics Department, St. Petersburg State Polytechnic University, 29 Polytechnicheskaya, St. Petersburg 195251, Russia \\ ${ }^{2}$ Solid State Electronic Sector, Ioffe Physical Technical Institute, 26 Polytechnicheskaya, St. Petersburg 194021, Russia \\ Correspondence should be addressed to E. V. Orlenko, eorlenko@mail.ru
}

Received 19 September 2010; Revised 21 December 2010; Accepted 14 February 2011

Academic Editor: Lowell D. Kispert

Copyright ( 2011 E. V. Orlenko et al. This is an open access article distributed under the Creative Commons Attribution License, which permits unrestricted use, distribution, and reproduction in any medium, provided the original work is properly cited.

\begin{abstract}
A new methodology of binding energy calculation with respect to different spin arrangements for a multiatomic electron system is developed from the first principle in the frame of the exchange perturbation theory (EPT). We developed EPT formalism in the general form of the Rayleigh-Srchödinger expansion with a symmetric Hamiltonian, taking into account an exchange and nonadditive contributions of a superexchange interaction. The expressions of all corrections to the energy and wave function were reduced to the nonsymmetric Hamiltonian form. The EPT method is extended for the case of degeneracy in the total spin of a system. As an example of the application of the developed EPT formalism for the degeneracy case, spin arrangements were considered for the key $\langle\mathrm{Mn}\rangle-\mathrm{O}-\langle\mathrm{Mn}\rangle\left(\langle\mathrm{Mn}\rangle: \mathrm{Mn}^{3+}\right.$ or $\left.\mathrm{Mn}^{4+}\right)$ fragments in manganites. In $\langle\mathrm{Mn}\rangle-\mathrm{O}-\left\langle\mathrm{Mn} \mathrm{for}_{\mathrm{La}} \mathrm{a}_{1 / 3} \mathrm{Ca}_{2 / 3} \mathrm{MnO}_{3}\right.$ are in good agreement the obtained estimations of Heisenberg parameter and binding energy with the available experimental data.
\end{abstract}

\section{Introduction}

Analytical calculation of the interaction potential in multiatomic electron systems with intermediate interatomic distances is essentially a problem in quantum chemistry. Intermediate distances indicate the distances on which interatomic interaction becomes small enough to be considered as a perturbation, with the exchange contribution being exponentially small, but exceeding van der Waals interaction. The quantum chemical analysis of a multiatomic system for the intermediate interatomic distances with respect to electronic spin ordering should definitely take into account intercenter electronic permutations.

The most efficient method for such distances is the perturbation theory method, because of its possibility to present the result in the form of expansion, each term of which has definite physical interpretation $[1,2]$. The variation procedures of atom and molecule wave function calculation or density functional approximation (DFT) method [3] in the frame of the mean field theory allow us to calculate pair potential of the particle interaction for the ground state. However, a common procedure in this case is forcing the wave function parameters to fit the experimental form of the potential $[1,2]$. Excited state determination needs additional convergence conditions. There is a quantum chemical Hartree-Fock method based on the Slater determinant, where antisymmetric wave function uses definite electron spin $z$-projections. The disadvantage of this method is concerned with the impossibility of determining the total spin value of the electronic system involved in interaction because of the noncommutating of the total spin quadrate operator of the system $\hat{S}^{2}$ and $\hat{s}_{i z}$ operators of the electronic spin $z$-projection for each particle, for example, $\left[\hat{S}^{2}, \hat{s}_{i z}\right] \neq 0$. It is important that interatomic potentials in the analytical form be found from the first principles, and exchange perturbation theory method gives such an opportunity. However, constructing the exchange perturbation theory formalism is connected with the two main following problems $[1,2]$.

First, the base of multiatomic system wave functions, being antisymmetric in intercenter permutations, is nonorthogonal and hence overcompleted (this is known as the overcompleteness catastrophe).

Second, the perturbation operator and unperturbed part of the Hamiltonian with respect to electron intercenter 
permutations are asymmetric. The total Hamiltonian $\hat{H}=$ $\hat{H}_{0}+\hat{V}$ of the system includes two terms: that of unperturbed Hamiltonian $\hat{H}_{0}$ of the noninteracting multiatomic system and the $\hat{V}$ perturbation, describing all electron and nuclei intercenter interactions. The total Hamiltonian of the system is symmetric with respect to intercenter permutations. In other words, $[\hat{H}, \hat{A}]=0$, but $\left[\hat{H}_{0}, \hat{A}\right] \neq 0$ and $[\hat{V}, \hat{A}] \neq 0$, where $\hat{A}$ is the antisymmetrization operator.

It is also necessary we take into account nonadditive superexchange contributions to the system energy and include pairwise nonadditive interactions in a symmetric Hamiltonian formalism. The constants of the superexchange interaction are usually estimated by combinations of pair integrals $[4,5]$, which actually means that the specific nonadditive part of multicenter interaction is discarded.

There are many formal EPT variants classified according to the way of overcoming these problems (a detailed description of this classification can be found in Kaplan's monograph $[1,2])$. All EPT variants can be divided into two groups, the first of which consisting of theories with non-symmetric Hamiltonian known as symmetry-adapted perturbation theories, for example [6-9]. It uses the fundamental basis of the zeroth functions, which are nonsymmetric in intercenter electron permutations. These functions are eigenfunctions of non-symmetric Hamiltonian of the unperturbed system. Wave function antisymmetrization is done post factum in each interpolation step. Finally, this analysis requires the use of an additional variation procedure. The second group consists of approaches, allowing for the general Rayleigh Schrödinger form use by setting up a special zeroth symmetric Hamiltonian, for which the antisymmetric functions are eigenfunctions. An attempt to modify the Hamiltonian in such a way was made in $[10,11]$ (known as the Sternheimer procedure), which led to a non-Hermitian form of the total Hamiltonian and actually limited the use of the method only by the two-electron systems. The symmetric Hamiltonian treatment has been abandoned due to several unsolvable problems in this approach. The main problem is that the perturbation corrections have no welldefined meaning in the limit of complete basis set. In contrast, methods with non-symmetric Hamiltonian known as symmetry-adapted perturbation theories became the mainstream method for intermolecular interaction calculations.

The Ritchie EPT [12], where the specific projection operators $\Lambda_{p}$ to the subspace of wave functions, corresponding to the determined permutation $p$, were sophistically entered and employed in the ETP formalism in the Wigner form of expansion, can also be assigned to the second group. The explicit forms of these operators were not given. A similar way of the Hamiltonian symmetrized form construction was used in [13], where the perturbation theory in the Rayleigh-Schrödinger form was developed; however, the $\Lambda_{p}$ operators were not obtained analytically either. The symmetric nonperturbed Hamiltonian and symmetric form of the perturbation operator were developed from the first principles in [14-16] for the two-center system, but only the attempts of developing analytical forms of the projection operators were made.
In the present paper we have developed a general EPT form for the multiatomic electron system for the case of the nonorthogonal basis of spatial parts of the wave function antisymmetrized with respect to the intercenter electron permutations. Due to the one-to-one correspondence between the symmetries of the wave function spin and spatial parts we create an algorithm permitting to determine the total spin value for the system of electrons involved in interaction. The analytical form of the $\hat{\Lambda}$-projection operators and the symmetric form of the unperturbed Hamiltonian as well as that of the perturbation operator have been obtained. The resultant expressions of the corrections to the energy and wave functions were analytically reduced to the nonsymmetric perturbation form. Such simple form is more useful for the applications; nevertheless, it contains all exchange and superexchange inputs. The EPT formalism is extended for the case of degeneracy in the total spin for multiatomic electronic system. As an example of the EPT developed formalism application for the case of degeneracy we consider the spin arrangement problem in the key manganese $\langle\mathrm{Mn}\rangle-$ $\mathrm{O}-\langle\mathrm{Mn}\rangle$ fragments $\left(\langle\mathrm{Mn}\rangle: \mathrm{Mn}^{3+}\right.$ or $\left.\mathrm{Mn}^{4+}\right)$ in manganites. On the basis of the analytical equations obtained, calculation of the binding energy and splitting in the total spin system have been performed for the $\mathrm{La}_{1 / 3} \mathrm{Ca}_{2 / 3} \mathrm{MnO}_{3}$ compound. The estimations obtained are in good agreement with the available experimental data.

\section{Exchange Perturbation Theory}

Exchange interaction between closely spaced atoms involves participation of out-shell electrons of these atoms. These shells can include not only the outermost valence shell, but also inner shells adjacent to the last shell, providing an appreciable contribution to the overlap energy. In general, a total of $N>2$ electrons will participate in the interaction of each pair of atoms. The total system Hamiltonian can be indicated as a Hamiltonian $H_{0}$ of the independent atoms plus perturbation operator $V$ taking into account all interatomic interactions:

$$
\hat{H}=H^{0}+V \text {. }
$$

The spatial part of the zero approximation wave function

$$
\Phi\left(r_{1}, \ldots, r_{N}\right)=\prod_{\alpha} \psi_{\alpha}\left(r_{i}, \ldots, r_{j}\right)
$$

is a simple product of atomic wave functions. We may leave the wave function spin part, if the unperturbed Hamiltonian and the perturbation do not directly contain spin operators, such as relativistic inputs of the spin-orbit and the spin-spin interactions. The spatial eigenfunction $\Phi_{n}\left(r_{1}, \ldots, r_{N}\right)$ of the unperturbed Hamiltonian

$$
H^{0} \Phi_{n}\left(r_{1}, \ldots, r_{N}\right)=E_{n}^{0} \Phi_{n}\left(r_{1}, \ldots, r_{N}\right),
$$

being antisymmetrized in intercenter permutations $\Psi_{n}^{0}\left(r_{1}, \ldots, r_{N}\right)=\hat{A} \Phi_{n}\left(r_{1}, \ldots, r_{N}\right)$, will not be an eigenfunction of the $H^{0}$ Hamiltonian. Here, $\hat{A}$ is the antisymmetrization operator. The antisymmetric wave function 
corrections calculated in the non-symmetric perturbation $V$ will contain nonphysical contributions [1].

The spatial part of the wave function antisymmetrized with the Young operator $\omega_{p, t}^{[\lambda]}$, where $[\lambda]$ is the form of Young tableaux and $t$ is the number of the standard tableaux, can be written as [2]

$$
\begin{aligned}
\Psi_{n}^{0}\left(r_{1}, \ldots, r_{N}\right) & =\hat{A} \Phi_{n}\left(r_{1}, \ldots, r_{N}\right) \\
& =\sum_{t} \omega_{p=0, t}^{[\lambda]} \Phi_{n}^{p=0} \\
& =\frac{1}{f_{n}^{P}} \sum_{p=0}^{P}(-1)^{g_{p}} \Phi_{n}^{p}\left(r_{1}, \ldots, r_{N}\right),
\end{aligned}
$$

where $p$ is the number of the permutation, $g_{P}$ is the permutation parity factor, and $\Phi_{n}^{p}\left(r_{1}, \ldots, r_{N}\right)$ is the same simple product as (2), but with the electron permutation of number $p$, where $P$ is the total number of the possible permutations, $1 / f_{n}^{P}$ is normalization $f_{n}^{P}=\sum_{p=0}^{P}(-1)^{g_{P}}\left(\Phi_{n}^{0} \mid \Phi_{n}^{p}\right)$. We will use the notation for the non-symmetric state vector- $\left.\mid \Phi_{n}^{p}\right)$ and for the antisymmetric state vector- $\left|\Psi_{n}^{0}\right\rangle$. Here is the normalization condition $\left(\Phi_{n}^{0}\left|\Psi_{n}^{0}\right\rangle=1\right.$, which differs by a factor $\sqrt{P}$ from the commonly used condition $\left\langle\Psi^{0} \mid \Psi^{0}\right\rangle=1$.

First, we will construct a symmetric form of the unperturbed Hamiltonian. The function $\Phi_{n}^{p}\left(r_{1}, \ldots, r_{N}\right)$ in which electrons have been interchanged is also an eigenfunction of the unperturbed system Hamiltonian $H_{P}^{0}$ with the same permutation, that is, $H_{P}^{0} \Phi_{i}^{P}=E_{i}^{0} \Phi_{i}^{P}$. Then we can write out the following set of equations:

$$
\begin{gathered}
\left.\left.H_{p=0}^{0} \mid \Phi_{i}^{p=0}\right)=E_{i}^{0} \mid \Phi_{i}^{p=0}\right), \\
\left.\left.H_{p=1}^{0} \mid \Phi_{i}^{p=1}\right)=E_{i}^{0} \mid \Phi_{i}^{p=1}\right), \\
\vdots \\
\left.\left.H_{P}^{0} \mid \Phi_{i}^{P}\right)=E_{i}^{0} \mid \Phi_{i}^{P}\right) .
\end{gathered}
$$

We sum up these equations so that, on the right, we obtain the function corresponding to the coordinate part of the Young tableaux, for which the symmetrization procedure is carried out in (4). The result is

$$
\left.\left.\sum_{p=0}^{p=P}(-1)^{g_{p}} H_{p}^{0} \mid \Phi_{i}^{p}\right)=E_{i}^{0} \sum_{p=0}^{p=P}(-1)^{g_{p}} \mid \Phi_{i}^{p=0}\right),
$$

or with respect to (4) we get the following expression:

$$
\left.\sum_{p=0}^{p=P}(-1)^{g_{p}} H_{p}^{0} \mid \Phi_{i}^{p}\right)=E_{i}^{0} f_{i}^{P}\left|\Psi_{i}^{0}\right\rangle .
$$

We introduce a projector $\left.\Lambda_{n}^{\pi}=\mid \Phi_{n}^{\pi}\right)\left(\Phi_{n}^{\pi} \mid\right.$ on the $\pi$-permutation vector of the state corresponding to energy level $E_{n}^{0}$. Applying it to the antisymmetrized vector $\left|\Psi_{n}^{0}\right\rangle$ with respect to the normalization condition we get

$$
\left.\Lambda_{n}^{\pi}\left|\Psi_{n}^{0}\right\rangle=(-1)^{g_{\pi}} \mid \Phi_{n}^{\pi}\right) .
$$

Using (8), equation (7) can be presented in the following form:

$$
\frac{1}{f_{i}^{P}} \sum_{p=0}^{p=P} H_{p}^{0} \Lambda_{i}^{p}\left|\Psi_{i}^{0}\right\rangle=E_{i}^{0}\left|\Psi_{i}^{0}\right\rangle,
$$

or

$$
\hat{H}_{0}\left|\Psi_{i}^{0}\right\rangle=E_{i}^{0}\left|\Psi_{i}^{0}\right\rangle,
$$

where the symmetrized unperturbed Hamiltonian has the form

$$
\hat{H}_{0}=\sum_{p=0}^{P} H_{p}^{0} \sum_{n} \frac{1}{f_{n}^{P}} \Lambda_{n}^{p}
$$

We can represent the perturbation operator in the symmetric form

$$
\widehat{V}=\sum_{p=0}^{P} V_{p} \sum_{n} \frac{1}{f_{n}^{P}} \Lambda_{n}^{p} .
$$

The total Hamiltonian of the interacting system is always invariant under the electron permutations so that the solution of the Schrödinger equation can be antisymmetric under the electron permutations

$$
\hat{H}|\Psi\rangle=E|\Psi\rangle,
$$

where $\hat{H}=\hat{H}_{0}+\hat{V}$ with respect to (11) and (12).

Solving (13) by the approximation method, we search for the perturbative corrections to the energy of the unperturbed system and the wave function (10). The perturbation operator in the form of (12) provides the same symmetry for the corrections as does the initial wave function. If interaction is small, we can represent the wave function and energy in the form of the following series:

$$
\begin{gathered}
\left|\Psi_{i}\right\rangle=\left|\Psi_{i}^{(0)}\right\rangle+\left|\Psi_{i}^{(1)}\right\rangle+\cdots, \\
E_{i}=E_{i}^{(0)}+E_{i}^{(1)}+\cdots
\end{gathered}
$$

In the first approximation in the perturbation, the Schrödinger equation takes the form

$$
\hat{H}_{0}\left|\Psi_{i}^{(1)}\right\rangle+\hat{V}\left|\Psi_{i}^{(0)}\right\rangle=E_{i}^{(1)}\left|\Psi_{i}^{(0)}\right\rangle+E_{i}^{(0)}\left|\Psi_{i}^{(1)}\right\rangle .
$$

The intermediate normalization condition is

$$
\left(\Phi_{i}\left|\Psi_{i}\right\rangle=\left(\Phi_{i}\left|\Psi_{i}^{0}\right\rangle,\right.\right.
$$

that is, $\left(\Phi_{i}\left|\left\{\left|\Psi_{i}\right\rangle-\left|\Psi_{i}^{0}\right\rangle\right\}=0\right.\right.$. Which means that all corrections to the vector $\left|\Psi_{i}^{0}\right\rangle$ of the zeroth approximation lie in the subspace orthogonal to this vector.

There is a projection operator $\hat{P}_{i}=\left|\Psi_{i}^{0}\right\rangle\left(\Phi_{i} \mid\right.$ in the subspace of vectors parallel to $\left|\Psi_{i}^{0}\right\rangle$.

Since

$$
\hat{P}_{i} \hat{H}_{0}\left|\Psi_{i}^{(1)}\right\rangle=E_{i}^{0} \hat{P}_{i}\left|\Psi_{i}^{(1)}\right\rangle
$$


is valid (see Appendix A), applying the projection operator $\widehat{P}_{i}$ to (15) results in $\left(\Phi_{i}|\hat{V}| \Psi_{i}^{0}\right\rangle\left|\Psi_{i}^{0}\right\rangle=E_{i}^{(1)}\left|\Psi_{i}^{0}\right\rangle$. Then the firstorder correction to the energy is

$$
E_{i}^{(1)}=\left(\Phi_{i}|\hat{V}| \Psi_{i}^{(0)}\right\rangle .
$$

Now we introduce the projector

$$
\widehat{O}_{i}=1-\widehat{P}_{i}
$$

on the subspace of vectors orthogonal to the vector $\left|\Psi_{i}^{0}\right\rangle$. The first-order corrections to the antisymmetrized vector $\left|\Psi_{i}^{0}\right\rangle$ will have the following form of expansion:

$$
\left|\Psi_{i}^{(1)}\right\rangle=\sum_{n}^{\prime} C_{n}\left|\Psi_{n}^{(0)}\right\rangle
$$

where $n \neq i$. Inserting the expansion (20) to (15) and applying the projector (19) to the result, we obtain the following equation:

$$
\widehat{O}_{i} \hat{V}\left|\Psi_{i}^{(0)}\right\rangle=\sum_{n} C_{n}\left(E_{i}^{0}-E_{n}^{0}\right)\left|\Psi_{n}^{(0)}\right\rangle .
$$

Using the property of completeness $\left.\sum_{n} \mid \Phi_{n}^{p}\right)\left(\Phi_{n}^{p} \mid=\hat{I}\right.$ of the orthogonal basis of the non-symmetric zeroth functions, we can get the left-hand side of (21) as follows (the details can be found in Appendix A):

$$
\hat{O}_{i} \hat{V}\left|\Psi_{i}^{(0)}\right\rangle=\frac{1}{P} \sum_{n}^{\prime} f_{n}^{p}\left|\Psi_{n}^{(0)}\right\rangle\left(\Phi_{n}^{(0)}\left|\hat{O}_{i} \hat{V}\right| \Psi_{i}^{(0)}\right\rangle
$$

From (21) and (22) we obtain the first-order corrections to the wave vector

$$
\begin{aligned}
& \left|\Psi_{i}^{(1)}\right\rangle=\frac{1}{P} \sum_{n} f_{n}^{p} \frac{\left(\Phi_{n}^{(0)}\left|\hat{O}_{i} \hat{V}\right| \Psi_{i}^{(0)}\right\rangle}{\left(E_{i}^{0}-E_{n}^{0}\right)}\left|\Psi_{n}^{0}\right\rangle, \\
& \left.\left.\mid \Phi_{i}^{(1)}\right)=\frac{1}{P} \sum_{n} f_{n}^{p} \frac{\left(\Phi_{n}^{(0)}\left|\hat{O}_{i} \hat{V}\right| \Psi_{i}^{(0)}\right\rangle}{\left(E_{i}^{0}-E_{n}^{0}\right)} \mid \Phi_{n}^{0}\right) .
\end{aligned}
$$

Since $\left(\Phi_{i}\left|\hat{O}_{i} \hat{V}\right| \Psi_{i}^{(0)}\right\rangle=\left(\Phi_{i}\left|\left\{1-\left|\Psi_{i}\right\rangle\left(\Phi_{i} \mid\right\} \hat{V}\left|\Psi_{i}^{(0)}\right\rangle \equiv 0\right.\right.\right.$ and $\left(\Phi_{n} \mid \hat{O}_{i}=\left(\Phi_{n} \mid-\left(\Phi_{n}\left|\Psi_{i}^{(0)}\right\rangle\left(\Phi_{i} \mid \approx\left(\Phi_{n} \mid\right.\right.\right.\right.\right.$ (see Appendix A), we get the final expressions:

$$
\begin{aligned}
& \left|\Psi_{i}^{(1)}\right\rangle=\frac{1}{P} \sum_{n}^{\prime} f_{n}^{P} \frac{\left(\Phi_{n}^{(0)}|\hat{V}| \Psi_{i}^{(0)}\right\rangle}{\left(E_{i}^{0}-E_{n}^{0}\right)}\left|\Psi_{n}^{(0)}\right\rangle, \\
& \left.\left.\mid \Phi_{i}^{(1)}\right)=\frac{1}{P} \sum_{n}^{\prime} f_{n}^{P} \frac{\left(\Phi_{n}^{(0)}|\hat{V}| \Psi_{i}^{(0)}\right\rangle}{\left(E_{i}^{(0)}-E_{n}^{(0)}\right)} \mid \Phi_{n}^{(0)}\right) .
\end{aligned}
$$

Here the intermediate normalization condition $\left\{\left(\Phi_{i}^{(0)} \mid+\right.\right.$ $\left(\Phi_{i}^{(1)} \mid\right\}\left\{\left|\Psi_{i}^{(0)}\right\rangle+\left|\Psi_{i}^{(1)}\right\rangle\right\}=1$ is used.

Using (9), (11), and (12), it can be shown that the integral $\left(\Phi_{n}^{(0)}|\hat{V}| \Psi_{i}^{(0)}\right\rangle$ is equal to the integral $\left\langle\Psi_{n}^{(0)}\left|V_{p=0}\right| \Phi_{i}^{(0)}\right)$, where perturbation $V$ has the simple non-symmetric form corresponding to the number of permutations $p=0$. Then (18) and (24) can be rewritten in the form of non-symmetric perturbation operator:

$$
E_{i}^{(1)}=\left\langle\Psi_{i}^{(0)}\left|V_{p=0}\right| \Phi_{i}\right)
$$

$$
\begin{aligned}
& \left|\Psi_{i}^{(1)}\right\rangle=\frac{1}{P} \sum_{n}^{\prime} f_{n}^{P} \frac{\left\langle\Psi_{n}^{(0)}\left|V_{p=0}\right| \Phi_{i}^{(0)}\right)}{\left(E_{i}^{(0)}-E_{n}^{(0)}\right)}\left|\Psi_{n}^{(0)}\right\rangle, \\
& \left.\left|\Phi_{i}^{(1)}\right\rangle=\frac{1}{P} \sum_{n}^{\prime} f_{n}^{P} \frac{\left\langle\Psi_{n}^{(0)}\left|V_{p=0}\right| \Phi_{i}^{(0)}\right)}{\left(E_{i}^{(0)}-E_{n}^{(0)}\right)} \mid \Phi_{n}^{(0)}\right) .
\end{aligned}
$$

Higher-order corrections are found in a similar way. So, for the second order we obtain the following equation:

$$
\begin{aligned}
& \hat{H}_{0}\left|\Psi_{i}^{(2)}\right\rangle+\hat{V}\left|\Psi_{i}^{(1)}\right\rangle \\
& \quad=E_{i}^{(0)}\left|\Psi_{i}^{(2)}\right\rangle+E_{i}^{(1)}\left|\Psi_{i}^{(1)}\right\rangle+E_{i}^{(2)}\left|\Psi_{i}^{(0)}\right\rangle,
\end{aligned}
$$

from which, after applying the operator $\widehat{P}_{i}$, we find the second-order correction to the energy:

$$
E_{i}^{(2)}=\sum_{k}^{\prime} \frac{f_{k}^{P}}{P} \frac{\left(\Phi_{i}^{(0)}|\hat{V}| \Psi_{k}^{0}\right\rangle\left(\Phi_{k}^{(0)}|\hat{V}| \Psi_{i}^{(0)}\right\rangle}{\left(E_{i}^{(0)}-E_{k}^{(0)}\right)} .
$$

Hence for the simple non-symmetric form it is

$$
E_{i}^{(2)}=\sum_{k}^{\prime} \frac{f_{k}^{P}}{P} \frac{\left\langle\Psi_{i}^{(0)}\left|V_{p=0}\right| \Phi_{k}^{(0)}\right)\left\langle\Psi_{k}^{(0)}\left|V_{p=0}\right| \Phi_{i}^{(0)}\right)}{\left(E_{i}^{(0)}-E_{k}^{(0)}\right)} .
$$

Similarly, applying the operator $\hat{O}_{i}$, we find the wave vector correction:

$$
\begin{aligned}
& \left|\Psi_{i}^{(2)}\right\rangle \\
& =\sum_{n}^{\prime} \sum_{k}^{\prime} \frac{f_{n}^{P} f_{k}^{P}}{P^{2}}\left|\Psi_{n}^{0}\right\rangle \frac{\left\langle\Psi_{n}^{0}\left|V_{p=0}\right| \Phi_{k}^{0}\right)\left\langle\Psi_{k}^{0}\left|V_{p=0}\right| \Phi_{i}^{0}\right)}{\left(E_{i}^{0}-E_{k}^{0}\right)\left(E_{i}^{0}-E_{n}^{0}\right)} \\
& \quad-\sum_{n}^{\prime}\left(\frac{f_{n}^{P}}{P}\right)^{2}\left|\Psi_{n}^{0}\right\rangle \frac{\left\langle\Psi_{n}^{0}\left|V_{p=0}\right| \Phi_{i}^{0}\right)\left\langle\Psi_{i}^{0}\left|V_{p=0}\right| \Phi_{i}^{0}\right)}{\left(E_{i}^{0}-E_{n}^{0}\right)^{2}} \\
& \quad-\frac{\left|\Psi_{i}^{0}\right\rangle}{2} \sum_{n}^{\prime}\left(\frac{f_{n}^{P}}{P}\right)^{2} \frac{\left\langle\Psi_{i}^{0}\left|V_{p=0}\right| \Phi_{n}^{0}\right)\left\langle\Psi_{n}^{0}\left|V_{p=0}\right| \Phi_{i}^{0}\right)}{\left(E_{i}^{0}-E_{n}^{0}\right)^{2}} \\
& \left.\mid \Phi_{i}^{(2)}\right) \quad \\
& \left.=\sum_{n}^{\prime} \sum_{k}^{\prime} \frac{f_{n}^{P} f_{k}^{P}}{P^{2}} \mid \Phi_{n}^{0}\right) \frac{\left\langle\Psi_{n}^{0}\left|V_{p=0}\right| \Phi_{k}^{0}\right)\left\langle\Psi_{k}^{0}\left|V_{p=0}\right| \Phi_{i}^{0}\right)}{\left(E_{i}^{0}-E_{k}^{0}\right)\left(E_{i}^{0}-E_{n}^{0}\right)}
\end{aligned}
$$




$$
\begin{aligned}
& \left.-\sum_{n}^{\prime}\left(\frac{f_{n}^{P}}{P}\right)^{2} \mid \Phi_{n}^{0}\right) \frac{\left\langle\Psi_{n}^{0}\left|V_{p=0}\right| \Phi_{i}^{0}\right)\left\langle\Psi_{i}^{0}\left|V_{p=0}\right| \Phi_{i}^{0}\right)}{\left(E_{i}^{0}-E_{n}^{0}\right)^{2}} \\
& -\frac{\left.\mid \Phi_{i}^{0}\right)}{2} \sum_{n}^{\prime}\left(\frac{f_{n}^{P}}{P}\right)^{2} \frac{\left\langle\Psi_{i}^{0}\left|V_{p=0}\right| \Phi_{n}^{0}\right)\left\langle\Psi_{n}^{0}\left|V_{p=0}\right| \Phi_{i}^{0}\right)}{\left(E_{i}^{0}-E_{n}^{0}\right)^{2}} .
\end{aligned}
$$

Here we used the intermediate normalization condition

$$
\left\{\left(\Phi _ { i } ^ { 0 } \left|+\left(\Phi _ { i } ^ { ( 1 ) } \left|+\left(\Phi_{i}^{(2)} \mid\right\}\left\{\left|\Psi_{i}^{0}\right\rangle+\left|\Psi_{i}^{(1)}\right\rangle+\left|\Psi_{i}^{(2)}\right\rangle\right\}=1 .\right.\right.\right.\right.\right.
$$

For the third-order correction to the energy, we get the following equation:

$$
\begin{aligned}
E_{i}^{(3)} & =\sum_{n}^{\prime} \sum_{k}^{\prime}\left(\frac{f_{n}^{P} f_{k}^{P}}{P^{2}}\right. \\
& \times \frac{\left\langle\Psi_{i}^{0}\left|V_{p=0}\right| \Phi_{k}^{0}\right)\left\langle\Psi_{k}^{0}\left|V_{p=0}\right| \Phi_{n}^{0}\right)\left\langle\Psi_{n}^{0}\left|V_{p=0}\right| \Phi_{i}^{0}\right)}{\left(E_{n}^{0}-E_{i}^{0}\right)\left(E_{k}^{0}-E_{i}^{0}\right)} \\
& -\left(\Phi^{0}{ }_{i}|\hat{V}| \Psi_{i}^{0}\right\rangle \\
& \left.\times \sum_{n}^{\prime} \frac{f_{n}^{P} f_{n}^{P}}{P^{2}} \frac{\left\langle\Psi_{i}^{0}\left|V_{p=0}\right| \Phi_{n}^{0}\right)\left\langle\Psi_{n}^{0}\left|V_{p=0}\right| \Phi_{i}^{0}\right)}{\left(E_{i}^{0}-E_{n}^{0}\right)^{2}}\right) .
\end{aligned}
$$

The set of the antisymmetrized wave function is nonorthogonal, but this set satisfies the property of completeness. Applying the operator $\sum_{n}\left|\Psi_{n}\right\rangle\left(\Phi_{n} \mid\right.$ to an arbitrary antisymmetrized vector $|\Psi\rangle$, we get

$$
\sum_{n}\left|\Psi_{n}\right\rangle\left(\Phi_{n}|\Psi\rangle=\sum_{n} \sum_{p=0}^{P} \mid \Phi_{n}^{p}\right)\left(\Phi_{n}|\Psi\rangle \cdot(-1)^{g_{p}} \frac{1}{f_{n}^{P}} .\right.
$$

Since this arbitrary vector $|\Psi\rangle$ is antisymmetric, it can be written out in the following form:

$$
\begin{aligned}
\sum_{n}\left|\Psi_{n}^{0}\right\rangle\left(\Phi_{n}|\Psi\rangle\right. & \left.=\sum_{n} \sum_{p=0}^{P} \frac{(-1)^{g_{p}}}{f_{n}^{P}} \mid \Phi_{n}^{p}\right)\left(\Phi_{n}|\Psi\rangle\right. \\
& \left.=\sum_{n} \sum_{p=0}^{P} \frac{(-1)^{g_{P}}}{f_{n}^{P}} \mid \Phi_{n}^{p}\right)\left(\Phi_{n}^{p}|\Psi\rangle(-1)^{g_{p}}\right. \\
& =\sum_{p=0}^{P} \frac{1}{f_{0}}|\Psi\rangle=\frac{P}{f_{0}}|\Psi\rangle,
\end{aligned}
$$

where $f_{n}^{P} \approx f_{0}=\sum_{p=0}^{p=P}\left(\Phi_{0} \mid \Phi_{0}^{p}\right)(-1)^{g_{p}}$ and $\left.\sum_{n} \mid \Phi_{n}^{p}\right)\left(\Phi_{n}^{p} \mid=\right.$ 1 are used. Then we get the equation

$$
\frac{f_{0}}{P} \sum_{n}\left|\Psi_{n}\right\rangle\left(\Phi_{n}\left|=\sum_{n}\right| \Phi_{n}\right)\left(\Phi_{n} \mid\right.
$$

This property of completeness (34) was proved with the use of two approximations:

$$
\begin{gathered}
\left(\Phi_{i}^{0} \mid \Phi_{n}^{p}\right) \ll\left(\Phi_{n}^{0} \mid \Phi_{n}^{p}\right) \sim\left(\Phi_{i}^{0} \mid \Phi_{i}^{p}\right), \\
f_{n}^{P} \approx f_{0}=\sum_{p=0}^{p=P}\left(\Phi_{0} \mid \Phi_{0}^{p}\right)(-1)^{g_{p}} .
\end{gathered}
$$

Thus, we have reduced the expressions for the high-order corrections to the energy and wave function for multiatomic system to the form of the exchange perturbation theory of Rayleigh-Schrödinger formalism with nonsymmetric Hamiltonian, so-called symmetry-adapted perturbation theories. In doing so the intercenter overlapping of the wave functions and superexchange effects were taken into account. The antisymmetrization with the Young tableaux makes the set of zero approach wave functions nonorthogonal; nevertheless, this set can meet the property of completeness under the condition of (35). The obtained EPT expansion is twoparametrical. The first small parameter is included in the perturbation, and it has the order of vanishing $a_{B} / R$, where $a_{B}$ is Bohr's radius, whereas $R$ is interatomic distance. The second parameter is the degree of interatomic overlapping, which has the order of vanishing $\exp \left(-R / a_{B}\right)$.

It should be underlined that for the intercenter distances $R>15 a_{B}$ the expressions for all corrections to the energy and wave function in the frame of EPT naturally become a standard expansion of Rayleigh-Schrödinger perturbation theory. In this case all exchange integrals containing the intercenter permutations $\left(\Phi^{\prime}|\hat{V}| \Phi\right) \ll(\Phi|\hat{V}| \Phi)$ are much less than the simple Coulomb contributions and the overlapping integrals $\left(\Phi \mid \Phi^{\prime}\right) \rightarrow 0$.

2.1. The Case of Degeneracy. The zeroth wave vector for a multicenter system can be antisymmetrized by various Young tableaux, which differ for different values of total system spin. In other words, a multiatomic system of noninteracting atoms is degenerate in total spin, with the degeneracy lifted by allowing for the ordinary intercenter Coulomb interaction. Thus, by the zeroth approximation we have

$$
\begin{gathered}
\left.\left|\Psi_{n \alpha}^{0}\right\rangle=A_{\alpha} \mid \Phi_{n}^{0}\right), \\
\hat{H}_{0 \alpha}\left|\Psi_{n \alpha}^{0}\right\rangle=E_{n}^{0}\left|\Psi_{n \alpha}^{0}\right\rangle, \\
\hat{H}_{0 \alpha}=\sum_{p=0, n}^{P} \frac{1}{f_{n}^{\alpha}} H_{p}^{0} \Lambda_{n}^{p},
\end{gathered}
$$

where $\left\{\left|\Psi_{n \alpha}^{0}\right\rangle\right\}$ is the set of wave vectors antisymmetrized by different Young tableaux $\alpha$ and corresponding to the same energy level $E_{n}^{0}$ of the system.

The wave vector of an interacting multicenter system of electrons is taken in the form

$$
\left|\Psi_{i}\right\rangle=\sum_{\beta} C_{\beta}^{0}\left|\Psi_{\beta i}^{0}\right\rangle+|\varphi\rangle
$$


where $\varphi$ is a wave function correction. If we substitute (37) in the complete Schrödinger equation (13), we obtain

$$
\begin{aligned}
\hat{H} \sum_{\beta} C_{\beta}^{0}\left|\Psi_{\beta}^{0}\right\rangle+\hat{H}|\varphi\rangle \\
\quad=\left(E_{i}^{0}+\varepsilon\right) \sum_{\beta} C_{\beta}^{0}\left|\Psi_{\beta}^{0}\right\rangle+\left(E_{i}^{0}+\varepsilon\right)|\varphi\rangle,
\end{aligned}
$$

where $\varepsilon$ is an energy correction. Since the total Hamiltonian is invariant under all permutations, it can be taken outside the summation sign in accordance with the Young tableau:

$$
\begin{aligned}
& \sum_{\beta}\left(\hat{H}_{\beta}^{0}+\hat{V}_{\beta}\right) C_{\beta}^{0}\left|\Psi_{\beta}^{0}\right\rangle+\hat{H}|\varphi\rangle \\
& \quad=\left(E_{i}^{0}+\varepsilon\right) \sum_{\beta} C_{\beta}^{0}\left|\Psi_{\beta}^{0}\right\rangle+\left(E_{i}^{0}+\varepsilon\right)|\varphi\rangle .
\end{aligned}
$$

Using (36), we can shift all terms containing the required wave function correction $\varphi$ to the left-hand side of (39) and all the other terms to the right-hand side. The result is

$$
\hat{H}|\varphi\rangle-\left(E_{i}^{0}+\varepsilon\right)|\varphi\rangle=\sum_{\beta}\left(\varepsilon-\hat{V}_{\beta}\right) C_{\beta}^{0}\left|\Psi_{\beta}^{0}\right\rangle .
$$

In (40) we drop all the terms whose order is higher than the first one, thus getting

$$
\left(\hat{H}_{\gamma}^{0}-E_{i}^{0}\right)|\varphi\rangle=\sum_{\beta}\left(\varepsilon-\hat{V}_{\beta}\right) C_{\beta}^{0}\left|\Psi_{\beta}^{0}\right\rangle .
$$

The solution of the homogeneous analog of (41) for $\varphi$ is $|\varphi\rangle=\left|\Psi_{\gamma}^{0}\right\rangle$. Then, according to the Fredholm alternative [17], the nonhomogeneous equation (41) has a solution only if the bra-vector $\left\langle\Psi_{\gamma}^{0}\right|$ is orthogonal to the entire right-hand side:

$$
\sum_{\beta}\left\{\varepsilon\left\langle\Psi_{\gamma}^{0} \mid \Psi_{\beta}^{0}\right\rangle-\left\langle\Psi_{\gamma}^{0}\left|\hat{V}_{\beta}\right| \Psi_{\beta}^{0}\right\rangle\right\} C_{\beta}^{0}=0
$$

Thus, we have a system of equations (42) for determining the coefficients $C_{\beta}^{0}$ of the regular zeroth wave function. The system has a solution only if the main determinant of (42) is equal to zero:

$$
\left|\varepsilon \Delta_{\gamma \beta}-\left\langle\Psi_{\gamma}^{0}\left|\hat{V}_{\beta}\right| \Psi_{\beta}^{0}\right\rangle\right|=0,
$$

where

$$
\Delta_{\gamma \beta}=\left\langle\Psi_{\gamma}^{0} \mid \Psi_{\beta}^{0}\right\rangle .
$$

This is the secular equation for determining the energy corrections. Because of

$$
\left\langle\Psi_{\gamma}^{0}\left|\hat{V}_{\beta}\right| \Psi_{\beta}^{0}\right\rangle=\Delta_{\gamma \beta}\left(\Phi^{0}\left|\hat{V}_{\beta}\right| \Psi_{\beta}^{0}\right\rangle
$$

the secular equation becomes

$$
\prod_{\beta=1}\left(\varepsilon-\left(\Phi^{0}\left|V_{\beta}\right| \Psi_{\beta}^{0}\right\rangle\right) \cdot \operatorname{det} \Delta_{\gamma \beta}=0 .
$$

Then for the corrections $\varepsilon$ to the energy we obtain definite expressions:

$$
\varepsilon=\left(\Phi^{0}\left|\hat{V}_{\alpha}\right| \Psi_{\alpha}^{0}\right\rangle
$$

or, in the simple non-symmetric form,

$$
\varepsilon=\left\langle\Psi_{\alpha}^{0}\left|\hat{V}_{p=0}\right| \Phi^{0}\right)
$$

only if the condition

$$
\operatorname{det} \Delta_{\alpha \beta} \neq 0
$$

is satisfied. Then the set of zeroth wave functions antisymmetrized by the Young tableaux is regular.

\section{Application of EPT to Manganites}

In the last decades many experiments have been devoted to the investigation of perovskites and manganites [1821]. Scientific interest to such systems has emerged due to their remarkable physical properties. Depending on the composition they show a variety of magnetic and electrical phenomena, including ferromagnetic, antiferromagnetic, charge, and orbital ordering. So, for example, the initial three-component $\mathrm{LaMno}_{3}$ and $\mathrm{AmnO}_{3}$ ( $\mathrm{A}: \mathrm{Sr}, \mathrm{Ca}$, or $\mathrm{Ba}$ ) are antiferromagnetic compounds with the spin moments located on the manganese ions. The four-component stoichiometric $\mathrm{La}_{1-x} \mathrm{~A}_{x} \mathrm{MnO}_{3}$ with $0.2 \leq x \leq 0.5$ is strongly ferromagnetic and demonstrates the metal-type conductivity below the Curie temperature, whereas the same system with $x>0.5$ is insulator with antiferromagnetic behavior below Neel temperature $T_{N}$.

Usually the microscopic description of magnetic ordering in crystalline structures including manganites [19-22] is either a statistical analysis of spin systems on the basis of the Heisenberg Hamiltonian or the analysis of magnetic susceptibility in the single-electron approximations in Stoner's [23-25], Hubbard's [4], Anderson's [23-25] or HohenbergKohn's type [3] models. The main parameter characterizing the spin system is the parameter of exchange (or superexchange) interaction. Hubbard-type model considers only the systems with electrons strongly localized at the centers. Therefore, the Coulomb exchange interaction in such system is calculated in the Wannier function approach. The use of a "truncated" Wannier-function basis artificially reduces the contribution of the intercenter exchange energy and, therefore, essentially eliminates the intercenter correlation effects. The Stoner model is used in the cases of nearly free electron gas. Anderson [23-25] was the first to point out the need for a more careful quantum chemical description of interatomic (interionic) interaction. Anderson's model considers the intracenter exchange (Hund's exchange) as well as intercenter exchange interactions; however, remaining in the frame of Hubbard's and Stoner's models, it does not take real superexchange interactions into account. As it was pointed out by Edio Dagotto [19-21], "the Heisenberg parameters $J$ usually have a large error and, as a consequence, it is common practice to simply consider them as free parameters to be determined from experiments." 
We will apply the EPT algorithm developed in the present work for the analysis of magnetic ordering and energy splitting for the key fragments of $\langle\mathrm{Mn}\rangle-\mathrm{O}-\langle\mathrm{Mn}\rangle(\langle\mathrm{Mn}\rangle$ : $\mathrm{Mn}^{3+}$ or $\mathrm{Mn}^{4+}$ ), which is critical for the interplay of charge, orbital, and spin ordering in manganites. On the basis of the energy splitting analysis we will estimate the main parameters of magnetic ordering (Heisenberg parameter and the resultant magnetic moment) for the $\mathrm{La}_{1 / 3} \mathrm{Ca}_{2 / 3} \mathrm{MnO}_{3}$ manganites.

Magnetic structures of $\mathrm{La}_{1-x} \mathrm{Ca}_{x} \mathrm{MnO}_{3}(x \geq 0.5)$ were determined from neutron diffraction measurements carried out by Wollan and Koehler [26]. According to [26], in the most studied $\mathrm{La}_{1 / 2} \mathrm{Ca}_{1 / 2} \mathrm{MnO}_{3}$ and $\mathrm{La}_{1 / 3} \mathrm{Ca}_{2 / 3} \mathrm{MnO}_{3}$ compounds magnetic structures belong to $\mathrm{CE}$ type. For instance, in $\mathrm{La}_{1 / 2} \mathrm{Ca}_{1 / 2} \mathrm{MnO}_{3}$ compound below $T_{\mathrm{N}} \approx 170 \mathrm{~K}$, it consists of two ferromagnetic zigzag $\left(\cdots \mathrm{Mn}^{3+}-\mathrm{Mn}^{4+}-\mathrm{Mn}^{3+}-\mathrm{Mn}^{4+} \cdots\right)$ chains coupled antiferromagnetically in the $(a-b)$ basal plane. These planes are stacked along the $c$-axis but with the opposite spins.

There are three possible three-center fragments in the key manganese chain, those of $\mathrm{Mn}^{3+}-\mathrm{O}^{2-}-\mathrm{Mn}^{4+}, \mathrm{Mn}^{4+}-\mathrm{O}^{2-}-$ $\mathrm{Mn}^{4+}$, and $\mathrm{Mn}^{3+}-\mathrm{O}^{2-}-\mathrm{Mn}^{3+}$. The magnetic form factors of the $\mathrm{Mn}^{4+}$ and $\mathrm{Mn}^{3+}$ ions correspond to the $3 d^{3}$ and $3 d^{4}$ states, respectively. For the $\mathrm{Mn}^{3+}-\mathrm{O}^{2-}-\mathrm{Mn}^{4+}$ fragment we consider the following microscopic model: owing to electrostatic dipole interaction, we assume that one electron of oxygen is drawn off from $\mathrm{O}^{2-}$ ion by $\mathrm{Mn}^{4+}$ resulting in the hybridization state of $2 p^{5}$ of oxygen and $3 d_{z^{2}}$ of manganese; then we get a virtual (or intermediate) $\left(\mathrm{Mn}^{3+}-\right.$ $\mathrm{O}^{-}+1 \mathrm{e} \leftrightarrow \mathrm{Mn}^{4+/ 3+}$ ) state. Due to the overlapping of the electron wave functions and their hybridization, the $\left(\mathrm{Mn}^{3+}-\right.$ $\mathrm{O}^{-}+1 \mathrm{e} \leftrightarrow \mathrm{Mn}^{4+/ 3+}$ ) fragment has a common electron cloud, making this fragment electrostatically stable with approximately identical two manganese ions with valence $3+$. The presence of significant $\mathrm{O}_{2 p}$ hole numbers in the manganites is confirmed experimentally $[27,28]$. Then in our calculations, because of the noted hybridization, we will take into account only three "active" electrons centred on the three different ions: $3 d_{z^{2}}, 3 d_{z^{2}}(\vec{r})+2 p^{5}(\vec{r}-\vec{R})$ for the manganese ions, and $2 p^{5}$ for the oxygen ion.

The complete electron wave function of the ion chain (for brevity, we denote this chain by I-II-III) can be antisymmetrized in three different ways corresponding to the

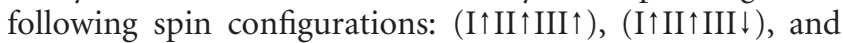
(I $\uparrow$ II $\downarrow$ III $\uparrow$ ). The corresponding Young tableaux are given in Figure 1.

At the beginning, the electrons with numbers 1,2, and 3 belong to the ions I, II, III, respectively. In this case the coordinate parts of the wave function corresponding to the Young tableaux $\alpha, \beta, \gamma$ are $\left[1^{3}\right],[2,1]$, and $[2,1]$ type, respectively:

$$
\begin{aligned}
& \Psi_{\alpha}^{0}\left(r_{1}, r_{2}, r_{3}\right) \\
& =\left(1-2 I_{1}^{2}+2 I_{2} I_{1}^{2}-I_{2}^{2}\right)^{-1}\left|\begin{array}{ccc}
\Psi_{\mathrm{I}}\left(r_{1}\right) & \Psi_{\mathrm{I}}\left(r_{2}\right) & \Psi_{\mathrm{I}}\left(r_{3}\right) \\
\Psi_{\mathrm{II}}\left(r_{1}\right) & \Psi_{\mathrm{II}}\left(r_{2}\right) & \Psi_{\mathrm{II}}\left(r_{3}\right) \\
\Psi_{\mathrm{III}}\left(r_{1}\right) & \Psi_{\mathrm{III}}\left(r_{2}\right) & \Psi_{\mathrm{III}}\left(r_{3}\right)
\end{array}\right|,
\end{aligned}
$$

Spin Young tableau

$(\alpha)$

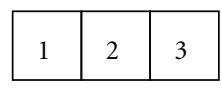

( $\beta)$

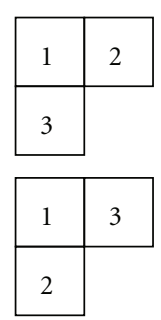

Coordinate Young tableau
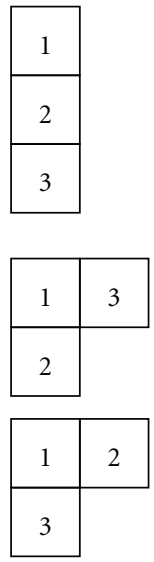

Figure 1

$$
\begin{aligned}
& \Psi_{\beta}\left(r_{1}, r_{2}, r_{3}\right)=\omega_{11}^{[21]} \Phi_{\mathrm{Mn}}\left(r_{1}\right) \Phi_{\mathrm{O}}\left(\frac{R}{2}-r_{2}\right) \Phi_{\mathrm{Mn}}\left(R-r_{3}\right), \\
& \Psi_{\gamma}\left(r_{1}, r_{2}, r_{3}\right)=\omega_{12}^{[21]} \Phi_{\mathrm{Mn}}\left(r_{1}\right) \Phi_{\mathrm{O}}\left(\frac{R}{2}-r_{2}\right) \Phi_{\mathrm{Mn}}\left(R-r_{3}\right),
\end{aligned}
$$

where, for instance, $r_{1}$ is the position vector of the electron 1 , $\Psi_{\mathrm{I}}\left(r_{1}\right)$ is a one electron wave function centered at the center I, and $R$ is the radius vector from the first manganese ion to the second one. The Young operator $\omega_{p t}^{[21]}$ of antisymmetrization for the tableau $[2,1]$ type is presented in Appendix B.

The integrals corresponding to the exchange densities are

$$
\begin{gathered}
I_{1}=\int \Psi_{\mathrm{I}}^{*}(r) \Psi_{\mathrm{II}}(r) d^{3} r=\int \Psi_{\mathrm{II}}^{*}(r) \Psi_{\mathrm{III}}(r) d^{3} r, \\
I_{2}=\int \Psi_{\mathrm{I}}^{*}(r) \Psi_{\mathrm{III}}(r) d^{3} r .
\end{gathered}
$$

For the initial distribution of the numbered electrons over the centers, the perturbation operator is

$$
\begin{aligned}
\hat{V}= & \frac{z_{1} z_{2} e^{2}}{\left|R_{\mathrm{I}}-R_{\mathrm{II}}\right|}+\frac{z_{1}^{2} e^{2}}{\left|R_{\mathrm{I}}-R_{\mathrm{III}}\right|}+\frac{z_{1} z_{2} e^{2}}{\left|R_{\mathrm{II}}-R_{\mathrm{III}}\right|}-\frac{z_{2} e^{2}}{\left|r_{1}-R_{\mathrm{II}}\right|} \\
& -\frac{z_{1} e^{2}}{\left|r_{1}-R_{\mathrm{III}}\right|}-\frac{z_{1} e^{2}}{\left|r_{2}-R_{\mathrm{I}}\right|}-\frac{z_{1} e^{2}}{\left|r_{2}-R_{\mathrm{III}}\right|}-\frac{z_{1} e^{2}}{\left|r_{3}-R_{\mathrm{I}}\right|} \\
& -\frac{z_{2} e^{2}}{\left|r_{3}-R_{\mathrm{II}}\right|}+\frac{e^{2}}{\left|r_{1}-r_{2}\right|}+\frac{e^{2}}{\left|r_{2}-r_{3}\right|}+\frac{e^{2}}{\left|r_{1}-r_{3}\right|} \\
= & \hat{V}_{\mathrm{I}, \mathrm{II}}+\hat{V}_{\mathrm{II}, \mathrm{III}}+\hat{V}_{\mathrm{III}, \mathrm{I}},
\end{aligned}
$$

where, for instance,

$$
\hat{V}_{\mathrm{I}, \mathrm{II}}=\frac{z_{1} z_{2} e^{2}}{\left|R_{\mathrm{I}}-R_{\mathrm{II}}\right|}-\frac{z_{2} e^{2}}{\left|r_{1}-R_{\mathrm{II}}\right|}-\frac{z_{1} e^{2}}{\left|r_{2}-R_{\mathrm{I}}\right|}+\frac{e^{2}}{\left|r_{1}-r_{2}\right|},
$$

describes the interaction of the ion cores I and II, the interaction of the electrons with the "foreign" nuclei, and the 
interaction of the electrons with each other, respectively. $R_{\mathrm{I}}$, $R_{\mathrm{II}}$, and $R_{\mathrm{III}}$ are position vectors of the interacting ions I, II, and III, respectively; $z_{1}, z_{2}$, and $z_{3}$ stand for the ion charges. Direct calculation yields the following values of the scalar product parameters:

$$
\begin{aligned}
& \Delta_{\alpha \beta}=\Delta_{\alpha \gamma}=0, \quad \Delta_{\beta \gamma}=\frac{2}{1-I_{2} I_{1}^{2}}, \quad \Delta_{\gamma \gamma}=\frac{4}{1-I_{2} I_{1}^{2}}, \\
& \Delta_{\alpha \alpha}=\frac{6}{1-2 I_{1}^{2}\left(1-I_{2}\right)-I_{2}^{2}}, \quad \Delta_{\beta \beta}=\frac{4}{1-I_{1}^{2}+I_{2}^{2}-I_{2} I_{1}^{2}} .
\end{aligned}
$$

The determinant condition is

$$
\left|\begin{array}{ccc}
\Delta_{\alpha \alpha} & 0 & 0 \\
0 & \Delta_{\beta \beta} & \Delta_{\beta \gamma} \\
0 & \Delta_{\gamma \beta} & \Delta_{\gamma \gamma}
\end{array}\right| \neq 0,
$$

Thus the condition to find a solution is met. In this case the energy corrections are

$$
\begin{aligned}
& \varepsilon_{\alpha}=\left\langle\Psi_{\alpha}^{0}\left|\hat{V}_{p=0}\right| \Phi^{0}\right), \\
& \varepsilon_{\beta}=\left\langle\Psi_{\beta}^{0}\left|\hat{V}_{p=0}\right| \Phi^{0}\right), \\
& \varepsilon_{\gamma}=\left\langle\Psi_{\gamma}^{0}\left|\hat{V}_{p=0}\right| \Phi^{0}\right),
\end{aligned}
$$

where

$$
\left.\left.\mid \Phi^{0}\right)=\mid \Phi_{\mathrm{Mn}}\left(r_{1}\right) \Phi_{O}\left(\frac{R}{2}-r_{2}\right) \Phi_{\mathrm{Mn}}\left(R-r_{3}\right)\right) .
$$

As an example, we consider the $\mathrm{La}_{1 / 3} \mathrm{Ca}_{2 / 3} \mathrm{MnO}_{3}$ manganite with the known magnetic ground state of $\mathrm{CE}$ type. For $\mathrm{La}_{1 / 3} \mathrm{Ca}_{2 / 3} \mathrm{MnO}_{3}$ due to Jahn-Teller effect, $R_{\mathrm{Mn}^{3+}-\mathrm{O}}=2.19 \AA$, $R_{\mathrm{Mn}^{4+}-\mathrm{O}}=1.97 \AA[18]$ in the zigzig chain, and then $\mid R_{\mathrm{I}}-$ $R_{\mathrm{II}}|=2.19 \AA,| R_{\mathrm{II}}-R_{\mathrm{III}} \mid=1.97 \AA$. Then the energy corrections calculated for all possible spin configurations in $\mathrm{Mn}^{3+}-\mathrm{O}^{2-}-\mathrm{Mn}^{4+}$ fragment are

$$
\begin{aligned}
& \varepsilon_{\alpha}(\uparrow \uparrow \uparrow)=-0.0165 \text { a.u, } \\
& \varepsilon_{\beta}(\uparrow \downarrow \downarrow)=-0.0173 \text { a.u, } \\
& \varepsilon_{\gamma}(\uparrow \downarrow \uparrow)=-0.0197 \text { a.u. }
\end{aligned}
$$

We note that the numerical values of the three-center superexchange contributions are of the same order as the pair couple exchange. They can yield the exchange energy correction of 10 to $30 \%$, depending on the factor $I_{1}, I_{2}$ accounting for the extent of the third electron overlap.

In the manganites, spin orientation according to inside Hund exchange $J_{\text {Hund }}=1.5 \mathrm{eV}[18]$ is ferromagnetic. For the considered fragment the magnetization and the Heisenberg parameter are $M=7 \mu_{B}$ and $J_{1} \approx \varepsilon_{\beta}-\varepsilon_{\gamma}=$ 0.0024 a.u. $=0.065 \mathrm{eV}$, correspondingly, with the mixing of the magnetization state $M=9 \mu_{B}$ and the $J_{1}^{\prime} \approx \varepsilon_{\beta}-\varepsilon_{\alpha}=$ 0.0008 a.u. $=-0.022 \mathrm{eV}$ parameter.

The $\mathrm{Mn}^{4+}-\mathrm{O}^{2-}-\mathrm{Mn}^{4+}$ fragment has an absolutely symmetric spatial form with respect to the intercenter and intracenter electron permutations as shown in Figure 2.

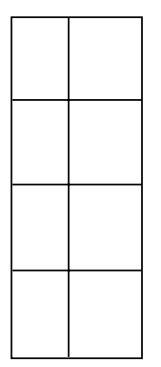

Figure 2

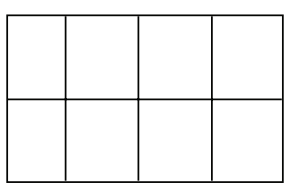

Figure 3

Its symmetry corresponding to the spin part is described by the only possible Young diagram (see Figure 3 ).

The total spin $S$ of such system equals zero and corresponds to the antiferromagnetic state. In the same way, for the $\mathrm{Mn}^{3+}-\mathrm{O} 2^{-}-\mathrm{Mn}^{3+}$ fragment we also obtain antiferromagnetic state with $S=0$. Then we estimate the Heisenberg parameter for the fragments under consideration. For $R=3.94 \AA$ [18] between two $\mathrm{Mn}^{4+}$ ions in the $(a-b)$ basal plane the estimation results in $J=$ $-0.0102 \mathrm{eV}$ corresponding to the antiferromagnetic state. For the $\mathrm{Mn}^{3+}-\mathrm{O} 2^{-}-\mathrm{Mn}^{3+}$ and $\mathrm{Mn}^{4+}-\mathrm{O} 2^{-}-\mathrm{Mn}^{4+}$ fragments located between two zigzag chains in the basal plane with $R=3.94 \AA$ [18] as well, we obtained $J_{\mathrm{Mn}^{3}-\mathrm{Mn}^{3}}=-0.0134 \mathrm{eV}$ and $J_{\mathrm{Mn}^{4}-\mathrm{Mn}^{4}}=-0.0102$, respectively. For the interplanar interactions in the $c$ direction both fragments have the absolutely symmetric spatial form with respect to intercenter and intracenter electron permutations, with the distance in this case being $R \approx 3.94 \AA$ [18] for both triads under considerations. Our estimations result in the following values

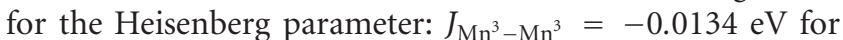
the $\mathrm{Mn}^{3+}-\mathrm{Mn}^{3+}$ fragment and $\mathrm{Jn}_{\mathrm{Mn}^{4}-\mathrm{Mn}^{4}}=-0.0102 \mathrm{eV}$ for the $\mathrm{Mn}^{4+}-\mathrm{Mn}^{4+}$ fragment. The antiferromagnetic character of exchange interaction obtained for these fragments is in agreement with the experimentally detected magnetic structure [26].

The estimation of the average Heisenberg $\langle J\rangle$ parameter with respect to degeneracy factor from (58) equals $\langle J\rangle \approx$ $0.04 \mathrm{eV}$; then the average magnetization per manganat ion is $\mu \approx 4.1 \mu_{B}$. From the statistic Heisenberg model [4] the Weiss temperature $\theta=(2 / 3) J$, then $\langle J\rangle \approx 0.023 \mathrm{eV}$. The estimated value of the Heisenberg parameter is in good agreement with $\theta \approx 175 \mathrm{~K}$ obtained in the experiment for the undoped compound [29].

\section{Conclusions}

The general EPT form for a multicenter electron system has been developed from the first principles, and the degree of approach has been presented for each order of expansion. 
The analytical form of the $\hat{\Lambda}$-projection operators and the symmetric form of the Hamiltonian unperturbed part and that of the perturbation operator have been obtained. In the frame of the exchange perturbation theory we have reduced the expressions for the high-order corrections to the energy and wave function to the standard form of the RayleighSchrödinger perturbation theory. The EPT formalism is extended for the degeneracy case in the total spin for the multicentered electronic system. This formalism does not need any additional variation procedure; each term of the expansion obtained has a certain physical interpretation. The contributions of superexchange interaction enter in the first-order terms. The main advantage of the presented formalism is the logical procedure of obtaining energy and wave function corrections, where it retains the one-to-one correspondence of the symmetry of spin and spatial parts of the wave function. Each energy correction corresponds to certain spin configuration. The presented ETP formalism is the only method allowing us to find the total spin value and spin configuration for the multicenter electron system.

As an example, using the developed EPT formalism with degeneracy in total spin, we obtained the analytical expressions of energy splitting for the key $\langle\mathrm{Mn}\rangle-\mathrm{O}-\langle\mathrm{Mn}\rangle$ fragments $\left(\langle\mathrm{Mn}\rangle: \mathrm{Mn}^{3+}\right.$ or $\left.\mathrm{Mn}^{4+}\right)$ in manganites. On the basis of these expressions the Heisenberg parameter and energy splitting were estimated for the $\mathrm{La}_{1 / 3} \mathrm{Ca}_{2 / 3} \mathrm{MnO}_{3}$ manganite. The Heisenberg parameter values obtained and the average magnetic moment of the system are in agreement with the known magnetic structure [26] and the experimentally obtained value of the Weiss temperature $[29,30]$ for the compound under consideration. The developed algorithm can be used for the analysis of Mn-site doping effect in manganites.

\section{Appendices}

\section{A. Details of the Theoretical Derivations}

Proof of (17), $\hat{P}_{i} \hat{H}_{0}\left|\Psi_{i}^{(1)}\right\rangle=E_{i}^{0} \hat{P}_{i}\left|\Psi_{i}^{(1)}\right\rangle$. One has

$$
\begin{aligned}
\hat{P}_{i} \hat{H}_{0}\left|\Psi_{i}^{(1)}\right\rangle & =\frac{\left|\Psi_{i}^{0}\right\rangle}{f_{i}^{P}} \sum_{p=0}^{P}\left(\Phi_{i}^{0}\left|H_{0}^{p}\right| \Phi_{i}^{p}\right)\left(\Phi_{i}^{p}\left|\Psi_{i}^{(1)}\right\rangle\right. \\
& =E_{i}^{0} \frac{\left|\Psi_{i}^{0}\right\rangle}{f_{i}^{P}} \sum_{p=0}^{P}\left(\Phi_{i}^{0} \mid \Phi_{i}^{p}\right)\left(\Phi_{i}^{p}\left|\Psi_{i}^{(1)}\right\rangle\right. \\
& =E_{i}^{0}\left|\Psi_{i}^{0}\right\rangle\left(\Phi_{i}^{0}\left|\Psi_{i}^{(1)}\right\rangle\right. \\
& =E_{i}^{0} \hat{P}_{i}\left|\Psi_{i}^{(1)}\right\rangle,
\end{aligned}
$$

where the completeness property $\left.\sum_{n} \mid \Phi_{n}^{p}\right)\left(\Phi_{n}^{p} \mid=\widehat{I}\right.$ is used.
The Properties of Projector on the Vector Subspace, Orthogonal to the Vector $\left|\Psi_{i}^{0}\right\rangle$. Since (1) $\hat{O}_{i}\left|\Psi_{i}^{0}\right\rangle \equiv 0$, (2) $\hat{O}_{i}\left|\Psi_{n}^{0}\right\rangle \approx\left|\Psi_{n}^{0}\right\rangle$, for the non-symmetric bra-vector:

$$
\begin{gathered}
\left(\Phi_{n} \mid \hat{O}_{i}=\left(\Phi_{n} \mid-\left(\Phi _ { n } | \Psi _ { i } ^ { 0 } \rangle \left(\Phi_{i} \mid \approx\left(\Phi_{n}\right),\right.\right.\right.\right. \\
\left(\Phi_{i} \mid \hat{O}_{i}=\left(\Phi_{i} \mid-\left(\Phi _ { i } | \Psi _ { i } ^ { 0 } \rangle \left(\Phi_{i} \mid \equiv 0 .\right.\right.\right.\right.
\end{gathered}
$$

Since $\left(\Phi_{i}^{0} \mid \Phi_{n}^{0}\right)=0$ and $\left(\Phi_{i}^{0} \mid \Phi_{n}^{p}\right) \ll\left(\Phi_{n}^{0} \mid \Phi_{n}^{p}\right) \approx\left(\Phi_{i}^{0} \mid \Phi_{i}^{p}\right)$ we have an expression:

$$
\begin{aligned}
P_{i}\left|\Psi_{n}^{0}\right\rangle & =\left|\Psi_{i}^{0}\right\rangle\left(\Phi_{i}^{0}\left|\Psi_{n}^{0}\right\rangle\right. \\
& =\left|\Psi_{i}^{0}\right\rangle \frac{1}{f_{P}} \sum_{p=0}^{P}\left(\Phi_{i}^{0} \mid \Phi_{n}^{p}\right)(-1)^{g_{p}} \approx 0 .
\end{aligned}
$$

Proof of (22), $\hat{O}_{i} \hat{V}\left|\Psi_{i}^{0}\right\rangle=1 / P \sum_{n}{ }^{\prime} f_{n}^{p}\left|\Psi_{n}^{0}\right\rangle\left(\Phi_{n}^{0}\left|\hat{O}_{i} \hat{V}\right| \Psi_{i}^{0}\right\rangle$.

One has

$$
\begin{aligned}
& \left.\frac{1}{P} \sum_{p=0}^{P} \sum_{n \neq i} \mid \Phi_{n}^{p}\right)\left(\Phi_{n}^{p}\left|\hat{O}_{i} \hat{V}\right| \Psi_{i}^{0}\right\rangle \\
& \left.=\frac{1}{P} \sum_{p=0}^{P} \sum_{n \neq i} \mid \Phi_{n}^{p}\right)(-1)^{p}\left(\Phi_{n}^{0}\left|\hat{O}_{i} \hat{V}\right| \Psi_{i}^{0}\right\rangle \\
& =\frac{1}{P} \sum_{n \neq i} f_{n}^{p}\left|\Psi_{n}^{0}\right\rangle\left(\Phi_{n}^{0}\left|\hat{O}_{i} \hat{V}\right| \Psi_{i}^{0}\right\rangle
\end{aligned}
$$

Proof of (44). One has

$$
\begin{aligned}
\left\langle\Psi_{\gamma}^{0}\left|\hat{V}_{\beta}\right| \Psi_{\beta}^{0}\right\rangle & =\frac{1}{f_{0}^{\gamma}} \sum_{p=0}^{P}(-1)^{g_{p y}}\left(\Phi^{p_{y}}\left|\hat{V}_{\beta}\right| \Psi_{\beta}^{0}\right\rangle \\
& =\frac{1}{f_{0}^{\gamma}} \sum_{p_{\gamma}=0}^{P}(-1)^{g_{p \gamma}+g_{p \beta}}\left(\Phi^{0}\left|\hat{V}_{\beta}\right| \Psi_{\beta}^{0}\right\rangle \\
& =\Delta_{\gamma \beta}\left(\Phi^{0}\left|\hat{V}_{\beta}\right| \Psi_{\beta}^{0}\right\rangle, \\
\Delta_{\gamma \beta} & =\left\langle\Psi_{\gamma}^{0} \mid \Psi_{\beta}^{0}\right\rangle=\frac{1}{f_{0}^{\gamma}} \sum_{p=0}^{P}(-1)^{g_{p}}\left(\Phi^{p_{\gamma}}\left|\Psi_{\beta}^{0}\right\rangle\right. \\
& =\sum_{p=0}^{P}(-1)^{g_{p \gamma}}\left(\Phi^{0}\left|\Psi_{\beta}^{0}\right\rangle\right. \\
& =\frac{1}{f_{0}^{\gamma}} \sum_{p=0}^{P}(-1)^{g_{p \gamma}+g_{p \beta} .} .
\end{aligned}
$$




\section{B. The Young Operators of Antisymmetrization}

Young Operators of Antisymmetrization for the Tableaus [2, 1] Type. Consider

$$
\begin{gathered}
\omega_{11}^{[21]}=\frac{1}{\sqrt{12}}\left(2+2 P_{12}-P_{23}-P_{13}-P_{123}-P_{132}\right), \\
\omega_{12}^{[21]}=\frac{1}{2}\left(P_{23}-P_{13}+P_{123}-P_{132}\right), \\
\omega_{21}^{[21]}=\frac{1}{2}\left(P_{23}-P_{13}-P_{123}+P_{132}\right), \\
\omega_{22}^{[21]}=\frac{1}{\sqrt{12}}\left(2-2 P_{12}+P_{23}+P_{13}-P_{123}-P_{132}\right) .
\end{gathered}
$$

Here the $P_{i j k}$ and $P_{i j}$ are operators of the numbered $j$ and $k$ electrons permutation.

The total antisymmetric function is

$$
\begin{aligned}
\Psi(1,2,3)= & \frac{1}{\sqrt{f_{\lambda}}} \sum_{r} \psi_{r}^{[\lambda]} \chi_{\tilde{r}}^{[\tilde{\lambda}]} \\
= & \left\{\omega_{11}^{[21]} \Phi_{\mathrm{Mn}}\left(r_{1}\right) \Phi_{O}\left(\frac{R}{2}-r_{2}\right) \Phi_{\mathrm{Mn}}\left(R-r_{3}\right)\right\} \\
& \otimes\left\{\omega_{22}^{[21]} \alpha 1 \beta 2 \alpha 3\right\} \\
& +\left\{\omega_{12}^{[21]} \Phi_{\mathrm{Mn}}\left(r_{1}\right) \Phi_{O}\left(\frac{R}{2}-r_{2}\right) \Phi_{\mathrm{Mn}}\left(R-r_{3}\right)\right\} \\
& \otimes\left\{\omega_{21}^{[21]} \alpha 1 \alpha 2 \beta 3\right\},
\end{aligned}
$$

where $\alpha 1$ and $\beta 2$ are spinors, corresponding to the particles numbered 1 and 2 .

\section{Acknowledgments}

F. E. Orlenko is grateful to the Science and High School Committee of St. Petersburg Government (Russia) for the Grant2010 for young scientists. This work was supported by the Ministry of Education and Science of the Russian Federation (Federal program "Scientific and Pedagogical Staff of Innovative Russia for 2009-2013"). The authors are thankful to Professor N. V. Popova of the Foreign Language Department of St. Petersburg State Polytechnic University for the help with language corrections.

\section{References}

[1] I. G. Kaplan, The Introduction to the Theory of Molecular Interactions, Nauka, Moskow, Russia, 1982.

[2] I. G. Kaplan, The Symmetry of the Many-Electronic Systems, Nauka, Moscow, Russia, 1969.

[3] W. Kohn and L. Sham, "Self-consistent equations including exchange and correlation effects," Physical Review, vol. 140, no. 4A, pp. A1133-A1138, 1965.

[4] R. M. White, Quantum Theory of Magnetism, Springer, Berlin, Germany, 1983.

[5] P. J. Hay, J. C. Thibeault, and R. Hoffmann, "Orbital interactions in metal dimer complexes," Journal of the American Chemical Society, vol. 97, no. 17, pp. 4884-4899, 1975.
[6] R. Eisenschitz and F. London, "Über das Verhältnis der van der Waalsschen Kräfte zu den homöopolaren Bindungskräften," Zeitschrift für Physik, vol. 60, no. 7-8, pp. 491-527, 1930.

[7] I. O. Hirschfelder and R. Silbey, "New type of molecular perturbation treatment," Journal of Chemical Physics, vol. 45, pp. 2188-2197, 1966.

[8] A. von der Avoird, "Perturbation theory for intermolecular interactions in the wave-operator formalism," Journal of Chemical Physics, vol. 47, pp. 3649-3653, 1967.

[9] I. Mayer, "A chemical energy component analysis," Chemical Physics Letters, vol. 332, no. 3-4, pp. 381-388, 2000.

[10] R. M. Sternheimer, "Electronic polarizabilities of ions from the Hartree-Fock wave functions," Physical Review, vol. 96, no. 4, pp. 951-968, 1954.

[11] S. T. Epstein and J. H. Kael, "Generalized sternheimer potential," Journal of Chemical Physics, vol. 44, no. 11, pp. 4347-4348, 1966.

[12] A. B. Ritchie, "Perturbation theory for exchange interactions between atoms and molecules. I," Physical Review, vol. 171, no. 1, pp. 125-127, 1968.

[13] A. A. Rumiantsev, "Perturbation theory with allowance for exchange forces," Soviet Physics—JETP, vol. 38, p. 459, 1976.

[14] E. V. Orlenko and A. A. Rumyantsev, "Perturbation theory with respect to intercenter electron exchange and superexchange with degeneracy," Soviet Physics—JETP, vol. 70, no. 7, p. 244, 1990.

[15] E. V. Orlenko and T. Yu. Latyshevskaya, "The method of exchange perturbation theory as applied to magnetic ordering in high-T materials," Journal of Experimental and Theoretical Physics, vol. 86, no. 6, pp. 1167-1176, 1998.

[16] E. V. Orlenko and B. G. Matisov, "Superexchange pairing and magnetic ordering in cuprate superconductors," Physics of the Solid State, vol. 41, no. 12, pp. 1951-1955, 1999.

[17] G. Korn and T. Korn, Mathematical Handbook, McGraw-Hill, New York, NY, USA, 2nd edition, 1968.

[18] V. M. Loktev and Yu. G. Pogorelov, "Peculiar physical properties and colossal magnetoresistance of manganites," Fizika Nizkikh Temperatur, vol. 26, no. 3, pp. 231-261, 2000 (Russian).

[19] E. Dagotto, T. Hotta, and A. Moreo, "Colossal magnetoresistant materials: the key role of phase separation," Physics Report, vol. 344, no. 1-3, pp. 1-153, 2001.

[20] E. Dagotto, Nanoscale Phase Separation and Collosal Magnetoresistance, Springer, Berlin, Germany, 2002.

[21] M. B. Salamon and M. Jaime, "The physics of manganites: structure and transport," Reviews of Modern Physics, vol. 73, no. 3, pp. 583-628, 2001.

[22] Y. Tokura, Ed., Colossal Magnetoresistance Oxides, Gordon and Breach, New York, NY, USA, 2000.

[23] P. W. Anderson, "A re-examination of concepts in magnetic metals: the 'nearly antiferromagnetic Fermi liquid' ," Advances in Physics, vol. 46, no. 1, pp. 3-11, 1997.

[24] P. W. Anderson, G. Baskaran, Z. Zou, and T. Hsu, "Resonating-valence-bond theory of phase transitions and superconductivity in $\mathrm{La}_{2} \mathrm{CuO}_{4}$-based compounds," Physical Review Letters, vol. 58, no. 26, pp. 2790-2793, 1987.

[25] E. C. Stoner, "Collective electron ferromagnetism," Proceedings of the Royal Society A, vol. 165, pp. 372-414, 1938.

[26] E. O. Wollan and W. C. Koehler, "Neutron diffraction study of the magnetic properties of the series of perovskite-type compounds $[(1-x) \mathrm{La}, x \mathrm{Ca}] \mathrm{MnO}_{3}, "$ Physical Review, vol. 100, no. 2, pp. 545-563, 1955. 
[27] J. M. D. Coey, M. Viret, and S. von Molnár, "Mixed-valence manganites," Advances in Physics, vol. 48, no. 2, pp. 167-293, 1999.

[28] H. L. Ju, H. C. Sohn, and K. M. Krishnan, "Evidence for $\mathrm{O}_{2 p}$ hole-driven conductivity in $\mathrm{La}_{1-x} \mathrm{Sr}_{x} \mathrm{MnO}_{3}(0 \leq x \leq 0.7)$ and $\mathrm{La}_{0.7} \mathrm{Sr}_{0.3} \mathrm{MnO}_{z}$ thin films," Physical Review Letters, vol. 79, no. 17, pp. 3230-3233, 1997.

[29] T. S. Orlova, J. Y. Laval, P. Monod et al., "Effect of Fe doping on structure, charge ordering, magnetic and transport properties of $\mathrm{La}_{0.33} \mathrm{Ca}_{0.67} \mathrm{Mn}_{1-y} \mathrm{Fe}_{y} \mathrm{O}_{3}(0 \leq y \leq 0.06)$," Journal of Physics: Condensed Matter, vol. 18, no. 29, pp. 6729-6748, 2006.

[30] T. S. Orlova, J. Y. Laval, Ph. Monod, P. Bassoul, J. G. Noudem, and E. V. Orlenko, "Influence of Mn-site doping on charge and orbital ordering in $\mathrm{La}_{1 / 3} \mathrm{Ca}_{2 / 3} \mathrm{Mn}_{1-y} \mathrm{M}_{y} \mathrm{O}_{3}$ manganites $(M=$ Ni,Ga)," Physical Review B, vol. 79, no. 13, Article ID 134407, 2009. 


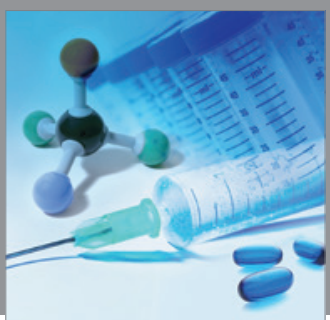

International Journal of

Medicinal Chemistry

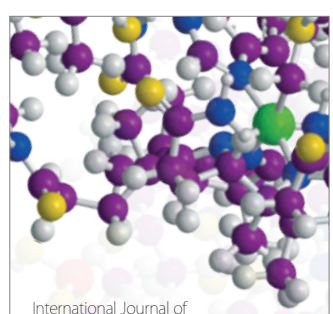

Carbohydrate Chemistry

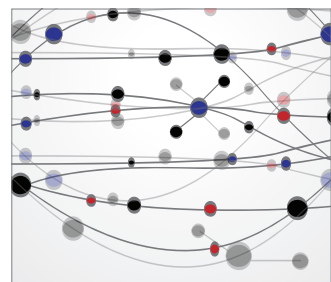

The Scientific World Journal
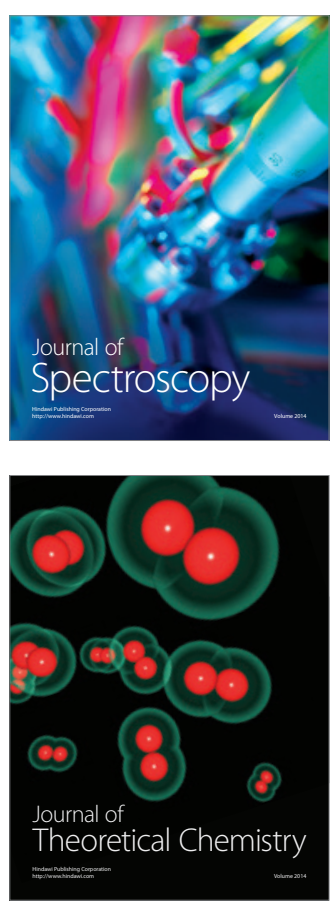
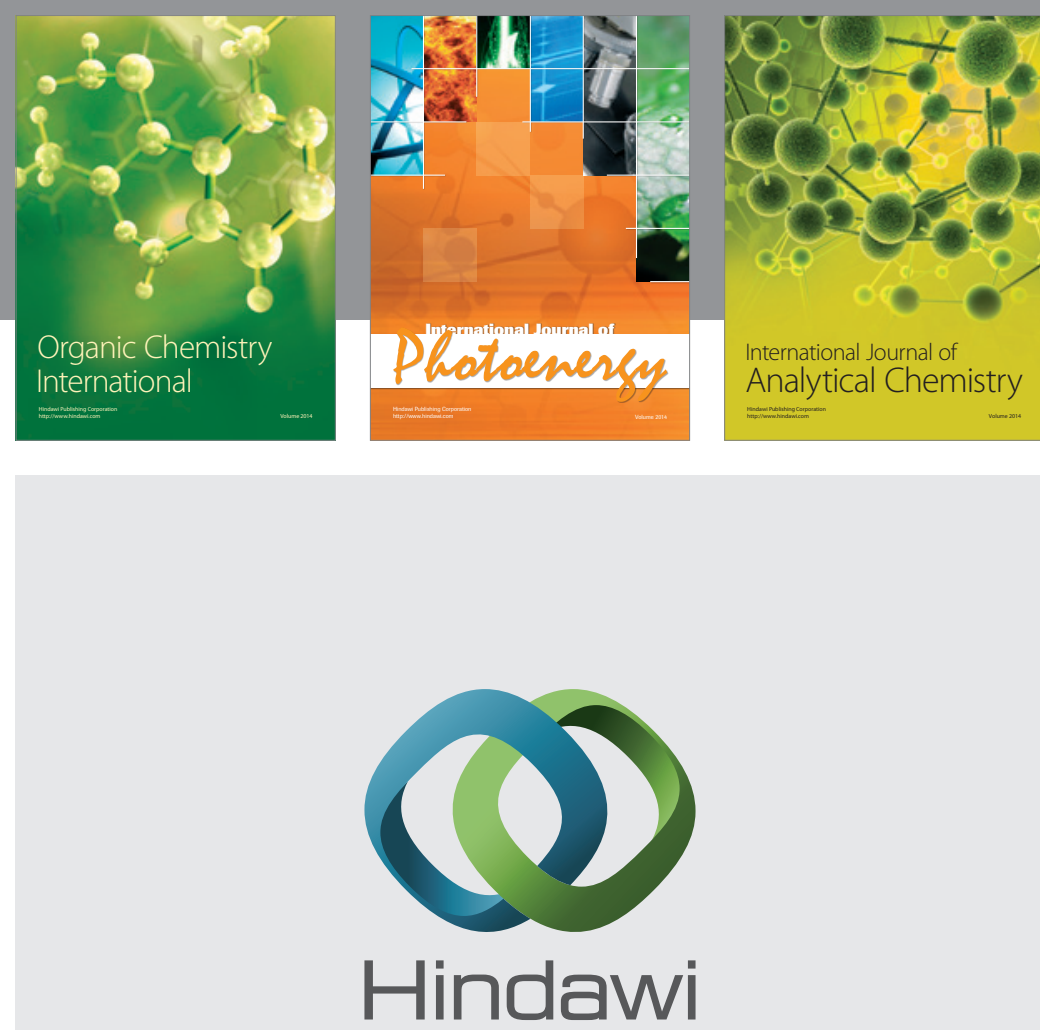

Submit your manuscripts at

http://www.hindawi.com
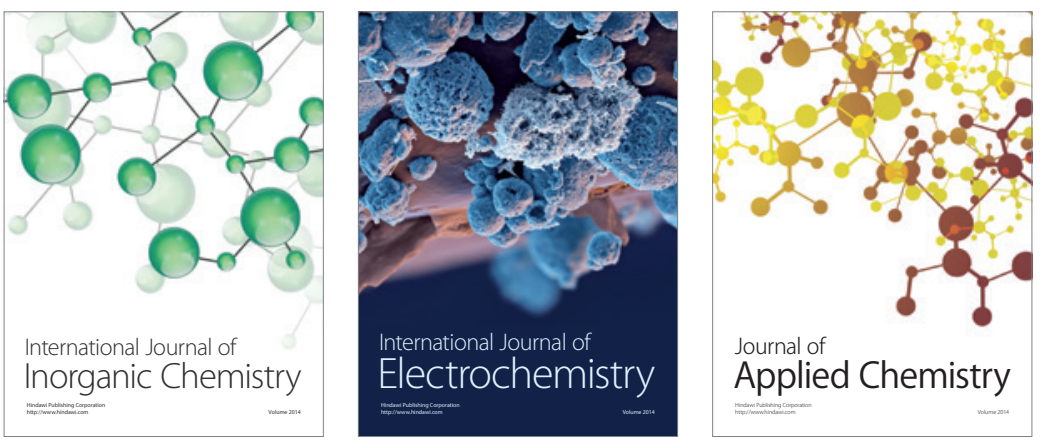

Journal of

Applied Chemistry
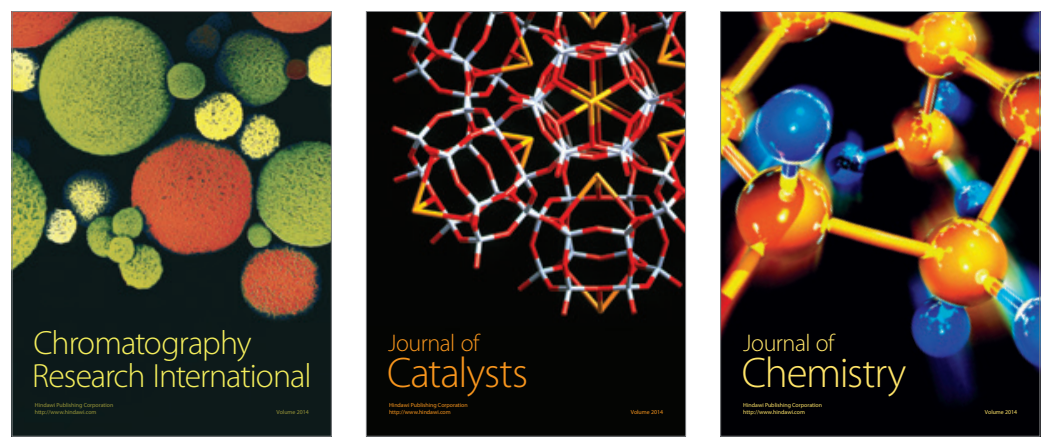
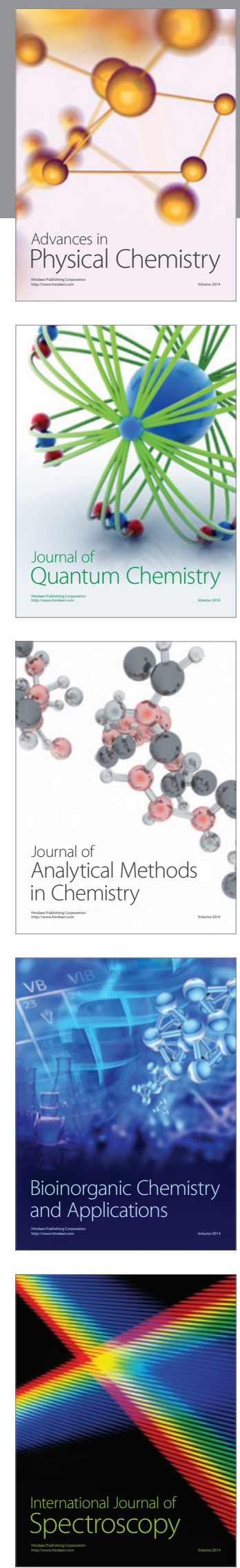